\title{
Gestión del riesǵo como eje articulador de un sistema de gestión integrado en las pymes
}

\section{Risk management as a hub articulator management system in SMEs}

Recibido: 30 de mavo de 2016

Martha Lucía Castañeda Muñoz***

Ecopetrol S.A.

Jairo Alexander Sánche* Prieto****

FTC Energy Group S.A

\section{RESUMEN}

En este artículo se presentan los resultados de una investigación realizada con el fin de reconocer la perspectiva de los auditores y coordinadores de sistemas de gestión sobre la aplicación del modelo de gestión del riesgo ISO 31000 y de aportar un modelo de gestión del riesgo que funcione como eje articulador y facilite la gestión integrada de las normas ISO 9001, ISO 14001 y OHSAS 18001, teniendo en cuenta los cambios futuros que tendrán estas normas. Para ello se realizaron encuestas a 26 auditores o consultores de sistemas de gestión y nueve entrevistas a coordinadores de sistemas de gestión en Pyme. El análisis de estas confirmó que las empresas objeto de estudio no realizan gestión del riesgo, no tienen en cuenta las partes interesadas ni el contexto donde existe la organización.

* Artículo resultado de investigación.

** Química y magíster (c) en Calidad y Gestión Integral. Analista de laboratorio en Ecopetrol S.A. Correo electrónico: marthapcastaneda@gmail.com

*** Ingeniero de Producción; especialista en Higiene y Salud Ocupacional y magíster (c) en Calidad y Gestión Integral. Jefe de División HSEQ en FTC Energy Group S.A. Correo electrónico: jairosanchez03@hotmail.com 
La investigación permitió identificar los elementos claves entre los sistemas de gestión ISO 9001, ISO 14001, OHSAS 18001 e ISO 31000, que contribuyen a que la gestión del riesgo se convierta en el eje articulador de estos sistemas de gestión y no en un cuarto sistema de gestión, lo que facilita la gestión integrada. Asimismo, el marco teórico y normativo, y los resultados del trabajo de campo aportaron significativamente a la definición del modelo de gestión del riesgo y su articulación e importancia con las otras normas.

Palabras clave: gestión del riesgo, gestión integrada, riesgo, sistema integrado de gestión.

\section{ABSTRACT}

In this paper the results of an investigation are presented to recognize the perspective of auditors and coordinators on the application of model risk management, ISO 31000 and provide a Risk Management Model function as linchpin and facilitate and integrated management of the ISO 9001, ISO 14001 and OHSAS 18001, taking into account future changes that these standards will have. surveys were conducted to 26 auditors and / or systems consultants management and 9 interviews to management systems coordinators in SMEs, which analysis confirmed that the companies under study does not perform risk management, not take into account the stakeholders and the context in which the organization exists.

The investigation identified the key elements between management systems ISO 9001, ISO 14001, OHSAS 18001 and ISO 31000, elements that contribute that the risk management will become the linchpin of these management systems and not in a room management system, providing the integrated management. Also, the framework theoretical and normative and results of fieldwork contributed significantly in the definition of risk management model and its articulation and importance with other standards.

Keywords: risk, management, integrated management, risk management system integral

\section{INTRODUCCIÓN}

Desde finales del siglo XX, se han advertido problemas de diferente índole en las organizaciones que han llevado a la Organización Internacional de Normalización (ISO) a desarrollar distintos modelos de gestión, como la norma ISO 9001 sobre sistemas de gestión de la calidad en las organizaciones (Atehortúa Hurtado, Bustamante Vélez y Valencia de los Ríos, Jorge Alberto, 2009). Asimismo, las preocupaciones por el deterioro del medio ambiente manifestadas en la Cumbre de Rio de Janeiro de 1992 alentaron el desarrollo del estándar ISO 14001 y los requerimientos normativos de la Organización Internacional del Trabajo (OIT) sobre seguridad y salud de los trabajadores motivaron la aparición del estándar OHSAS 18001 (Atehortúa Hurtado et al., 2009).

En el transcurso de la última década, el terrorismo, la crisis financiera global, los fenómenos meteorológicos extremos, la integración global de las empresas, junto con un creciente deseo de desarrollar e implementar procesos de gestión de riesgos consistentes en todo el mundo llevaron a reconocer la necesidad de una norma internacional y a la creación de la norma técnica ISO 31000:2009 (Woods, 2011), que es útil para todas las organizaciones, ya que puede ser usada no solo a nivel estratégico de la empresa, sino también a nivel operativo, independientemente de su tipo, sector y tamaño (Passenheim, 2010). 
Adicionalmente, la normatividad nacional e internacional, desde principios de los años noventa, ha definido la importancia de implementar este tipo de herramientas de control que permiten un mejor entendimiento de los procesos, anticiparse a los diferentes tipos de riesgo y facilitar la toma de decisiones gerenciales (Álvarez Uribe, Roca Garavito, Chaux Donado, Pineda Granados, 2012). En la tabla 1 se relacionan los principales contenidos de las normas que orientan los sistemas de gestión que conforman el modelo que se pretende construir en este estudio, es decir, ISO 9001, ISO 14001, OHSAS 18001 e ISO 31000. El tema de la gestión de riesgos aparece en todos los modelos de gestión normalizados, de manera explícita o implícita (Atehortúa Hurtado et al., 2009).

Tabla 1. Modelos de gestión, características y riesgos

\begin{tabular}{|c|c|c|c|}
\hline MODELO & $\begin{array}{l}\text { TIPO DE } \\
\text { SISTEMA }\end{array}$ & $\begin{array}{c}\text { FINALIDAD Y } \\
\text { CARACTERÍSTICAS }\end{array}$ & $\begin{array}{c}\text { TIPO DE RIESGO Y } \\
\text { ENFOQUE DEL RIESGO }\end{array}$ \\
\hline ISO 9001 & $\begin{array}{l}\text { Sistema de } \\
\text { Gestión de la } \\
\text { Calidad } \\
\text { (SGC) }\end{array}$ & $\begin{array}{l}\text { Dirigir y controlar la organización con respecto a la calidad. } \\
\text { Construido a partir de los conceptos de calidad, cliente, procesos } \\
\text { y gestión; hace énfasis en el cumplimiento de los requisitos } \\
\text { de conformidad del producto o servicio y en la satisfacción del } \\
\text { cliente. } \\
\text { Está basado en el ciclo planificar-hacer-verificar-actuar (PHVA) y } \\
\text { fundamentado en ocho principios básicos (Atehortúa et al., 2009). }\end{array}$ & $\begin{array}{l}\text { Incumplimiento de los requisitos de } \\
\text { calidad de los productos o servicios de } \\
\text { la organización. } \\
\text { El enfoque del riesgo está implícito } \\
\text { en los conceptos de no conformidad, } \\
\text { producto no conforme, acción } \\
\text { correctiva y acción preventiva }\end{array}$ \\
\hline ISO 14001 & $\begin{array}{l}\text { Sistema } \\
\text { de Gestión } \\
\text { Ambiental } \\
\text { (SGA) }\end{array}$ & $\begin{array}{l}\text { Desarrollar e implementar directrices y criterios que le permitan } \\
\text { a la organización gestionar sus aspectos ambientales, desarrollar } \\
\text { e implementar la política ambiental y ayudar a lograr metas } \\
\text { ambientales. } \\
\text { Basado en el ciclo PHVA, proporciona los elementos de un } \\
\text { SGA eficaz. No contiene un conjunto de principios declarados } \\
\text { explícitamente (Atehortúa et al., 2009). }\end{array}$ & $\begin{array}{l}\text { Afectación del medio ambiente como } \\
\text { resultado de las interacciones de la } \\
\text { organización con él. } \\
\text { El enfoque del riesgo está implícito en } \\
\text { los conceptos de aspecto ambiental e } \\
\text { impacto ambiental y en los planes de } \\
\text { emergencias. }\end{array}$ \\
\hline $\begin{array}{c}\text { OHSAS } \\
18001\end{array}$ & $\begin{array}{l}\text { Sistema de } \\
\text { Gestión de } \\
\text { Seguridad } \\
\text { Industrial } \\
\text { y Salud } \\
\text { Ocupacional } \\
\text { (SISO) }\end{array}$ & $\begin{array}{l}\text { Desarrollar e implementar directrices y criterios que le permitan } \\
\text { a la organización gestionar sus riesgos de seguridad y salud } \\
\text { ocupacional, desarrollar e implementar la política de SISO y } \\
\text { ayudar a lograr los objetivos de SISO. } \\
\text { Está basado en el ciclo PHVA, brinda los elementos de un } \\
\text { sistema de gestión eficaz. No contiene un conjunto de principios } \\
\text { declarados explícitamente (Atehortúa et al., 2009). }\end{array}$ & $\begin{array}{l}\text { Lesiones o enfermedades que } \\
\text { puedan ser causadas por eventos o } \\
\text { exposiciones peligrosas. } \\
\text { El enfoque del riesgo está explícito. }\end{array}$ \\
\hline ISO 31000 & $\begin{array}{l}\text { Sistema de } \\
\text { Gestión del } \\
\text { Riesgo } \\
\text { (SGR) }\end{array}$ & $\begin{array}{l}\text { Desarrollar e implementar directrices y criterios que le permitan } \\
\text { a la organización realizar una adecuada gestión en aquellos } \\
\text { aspectos negativos que tienen probabilidad de afectar el } \\
\text { cumplimiento de los propósitos institucionales. } \\
\text { Está basado en once principios que es necesario satisfacer para } \\
\text { hacer que la gestión del riesgo sea eficaz; requiere un marco de } \\
\text { referencia para la gestión del riesgo (Instituto Colombiano de } \\
\text { Normas Técnicas y Certificación, [Icontec] 2012). }\end{array}$ & $\begin{array}{l}\text { Afectación sobre áreas de impacto: } \\
\text { medio ambiente, personas, activos, } \\
\text { costos, ingresos, comunidad, etc. } \\
\text { El enfoque del riesgo está explícito. }\end{array}$ \\
\hline
\end{tabular}

Fuente: Elaboración propia basas en Atehortúa Hurtado et al. (2009). 
Actualmente, las pymes constituyen el sostén de muchas economías y aunque la globalización, el fácil acceso a los sistemas de gestión y las múltiples ventajas competitivas que trae la implementación de los sistemas en una organización contribuyan a que aumente el número de empresas certificadas en el mundo, es evidente que las pymes tienen dificultades en la aplicación de estos sistemas.
En la literatura muchos autores han hecho énfasis en la necesidad de integrar los sistemas de gestión en las organizaciones y han propuesto diferentes enfoques metodológicos de integración. En la tabla 2 se sintetizan los diferentes enfoques metodológicos planteados por algunos autores.

Tabla 2. Algunos enfoques metodológicos de integración

\begin{tabular}{|c|c|}
\hline AUTOR & ENFOQUE \\
\hline Bernardo, Karapetrovic y Casadesús & $\begin{array}{l}\text { Está basado en la compatibilidad de las normas en las que se apoyan los sistemas de gestión } \\
\text { normalizados de la calidad, medio ambiente y seguridad y salud en el trabajo. La norma ISO } 9001 \\
\text { tiene un claro enfoque de integración basada en procesos, pero las normas ISO } 14001 \text { y OHSAS } \\
18001 \text { tienen un enfoque de integración fundada en el ciclo de mejora continua. }\end{array}$ \\
\hline Wilkinson y Dale; Bobrek y Sokovic. & $\begin{array}{l}\text { Está soportado por un enfoque sistémico, en el que los elementos del sistema integrado se } \\
\text { disponen con el propósito de orientarse a la consecución de unos resultados esperados. Dentro } \\
\text { de este enfoque los procesos son considerados las unidades que permiten la transformación de } \\
\text { entradas en salidas. }\end{array}$ \\
\hline Labodová & $\begin{array}{l}\text { Está basado en un enfoque metodológico que considera el análisis de riesgos como pilar para la } \\
\text { integración, ampliando el concepto de riesgo desde una orientación exclusiva hacia la seguridad } \\
\text { y salud de los trabajadores, hacia la consideración de los riesgos asociados a los clientes y de los } \\
\text { riesgos relacionados con el entorno ambiental. Son los procesos la fuente de riesgos relacionados } \\
\text { con las diferentes partes interesadas con las que interactúan. }\end{array}$ \\
\hline Fresner y Engelhardt & Está basado en el análisis de procesos fundamentales de la organización. \\
\hline Norma UNE 66177:2005 & $\begin{array}{l}\text { La integración depende de los niveles de madurez en la gestión por procesos; considera la gestión } \\
\text { por procesos como mejor método para la integración de los sistemas de gestión. }\end{array}$ \\
\hline Ferguson Amores & $\begin{array}{l}\text { La base de los procesos es el método más eficaz frente a otras estrategias de integración, como la } \\
\text { integración por políticas o la integración cultural }\end{array}$ \\
\hline Zeng & $\begin{array}{l}\text { Los procesos por desarrollar deberían documentar la manera de llevar a cabo las actividades } \\
\text { necesarias para cumplir con la política de la organización, y esto se puede realizar considerando la } \\
\text { influencia de todas las áreas objeto de integración, desde una perspectiva de identificación de las } \\
\text { sinergias existentes entre las diferentes áreas. }\end{array}$ \\
\hline
\end{tabular}

Fuente: Carmona Calvo y Rivas Zapata, 2010. 
Hoy por hoy, existen diversas normas para la integración de sistemas como la norma AS/NZS 4581, la norma UNE 66177 y la PAS 99. La primera identifica los elementos comunes a los tres sistemas de gestión (calidad, medio ambiente y seguridad y salud en el trabajo) y aporta una visión de esos elementos, mientras que la segunda no trata de identificar dichos elementos ni de describir elementos similares, pero sí ofrece una metodología para que la propia organización identifique las sinergias considerando a los procesos como la vía para ello (Carmona Calvo, 2008). Por su parte, la tercera tiene como fin simplificar la implementación de múltiples normas del sistema y de cualquier evaluación del cumplimiento asociada (British Standards Institution, s. f. [BSI]).

En efecto, en estas actividades de integración las empresas y responsables de los sistemas de gestión recurren a las metodologías definidas, pero como concluyen entidades como la Fundación Mapfre, "la bibliografía científica sobre el campo de investigación de la integración de sistemas de gestión demuestra que éste todavía se encuentra en sus albores" (SánchezToledo Ledesma, s. f., s. p.). Los pocos estudios basan la integración en la identificación de los elementos comunes de las tres normas; las organizaciones toman como referencia las tablas de compatibilidad que cada una de estas normas aporta, para hacer la llamada integración de sistemas de gestión.

En Colombia, sobresalen los autores Castillo Pinzón y Martínez Tobo (2004), con su libro Enfoque para combinar e integrar la gestión de sistemas, en el que hacen referencia a tres enfoques diferentes para integrar los sistemas de gestión: enfoque por elementos de los modelos, enfoque por esquema administrativo funcional y enfoque operativo por procesos, siendo este último el recomendado por los autores. De igual forma, Atehortúa Hurtado et al. (2009), en su libro Sistema de gestión integral, una sola gestión un solo equipo, proponen realizar la integración utilizando un enfoque de procesos. En ambos libros, los autores coinciden en reconocer que la gestión es una sola y plantean que esta condición incluye aplicar un solo ciclo PHVA (planear - hacer-verificar-actuar).

Aunque las razones en las dificultades de integración son múltiples, según Torres Becerra y Suna Ladino (2012), el problema principal de las organizaciones en relación con los sistemas integrados de gestión "radica en que cada uno de estos modelos de gestión está enfocado en diferentes aspectos organizacionales, lo que contribuye a que estos sistemas carezcan de una adecuada integración de sus estrategias" (p. 20). Por lo tanto, y de acuerdo con las apreciaciones de Velásquez Rueda (2012): "las organizaciones terminan convirtiéndose en un rompecabezas que no se articula ni interrelaciona porque se hace énfasis en el cumplimiento de los requisitos sin establecer el vínculo con el sistema, lo que impide que se lleve a cabo una gestión integrada" (p. 14).

La integración de estos sistemas de gestión tiene que contribuir a satisfacer las necesidades que tienen las empresas para mejorar su competitividad y deben responder ante las nuevas condiciones con las que se desarrollan. Las tres normas, ISO 9001, ISO 14001 y OHSAS 18001, se desarrollan con un enfoque de prevención; cada uno de los requisitos descritos en las normas está asociado a un riesgo. Todas las empresas y sus respectivas áreas se encuentran inmersas en un diverso número de dificultades y es desde estas premisas de enfoque que la adopción de la gestión del riesgo como elemento articulador posibilita un marco amplio para implantar y mantener los sistemas mencionados. En este sentido, la gestión del riesgo tiene cada día más importancia, razón por la cual las empresas buscan conocer el nivel de riesgo en el que están incurriendo en las diferentes áreas; todas las actividades de una organización implican riesgo (Icontec, 2012) y la administración de riesgos "es un proceso reiterativo que puede contribuir a la mejora organizacional" (Jiménez, s. f., s. p.). 
Esta propuesta investigativa se convierte en una alternativa que aporta una propuesta metodológica de la cual se carece; además, fomenta la gestión integrada de los sistemas de calidad, medio ambiente y salud ocupacional, ya que las metodologías actualmente usadas, se reitera, presenta barreras y como resultado se han dado integraciones parciales que no tienen un impacto significativo sobre los sistemas.

El objetivo de esta investigación es diseñar un modelo de gestión del riesgo que le permita articular coherentemente los sistemas y facilite la gestión integrada en las pymes de cualquier sector económico, que tenga implementados los sistemas de gestión de ISO 9001, ISO 14001 y OHSAS 18001.

\section{METODOLOGÍA}

Esta es una investigación mixta porque integró datos cuantitativos y cualitativos con alcance descriptivo, dado que permitió realizar un diagnóstico sobre la perspectiva de los auditores y coordinadores sobre la gestión del riesgo en las pymes.

En este estudio se seleccionaron dos unidades de muestreo y se utilizaron muestras censales. La primera unidad de muestreo seleccionada fue una población de 26 auditores o consultores de sistemas de gestión en pymes a los que se les realizó una encuesta. La segunda unidad de muestreo seleccionada fueron coordinadores de sistemas de gestión de pymes, a los cuales se les hizo una entrevista, lo que constituye una población de nueve pymes certificadas en las normas ISO 9001, ISO 14001 y OHSAS 18001.

Con el ánimo de comprender las interacciones de los requisitos normativos, se realizó una matriz de correspondencia entre las normas técnicas ISO 9001, ISO 14001, OHSAS 18001 e ISO 31000. La matriz se comparó con el Anexo SL de ISO. De estas actividades se extrajeron los siguientes aspectos para el desarrollo de la investigación: la matriz y los elementos clave determinados se utilizaron como punto de partida para la elaboración de la encuesta y la entrevista usadas finalmente.

Para garantizar la validez de la encuesta y el guión de la entrevista se realizó para cada instrumento un estudio piloto. En relación con la confiabilidad de la encuesta, se aplicó el coeficiente alfa de Cronbach mediante el uso del software SPSS® (Statistical Package for the Social Sciences). Se llevó a cabo la triangulación de los métodos cuantitativos y cualitativos usados; posteriormente, se contrastó el marco referencial, la hipótesis y los resultados de las encuestas y entrevistas para establecer las conclusiones.

Como hipótesis se contempló la siguiente: la propuesta del modelo de gestión del riesgo se constituye en un eje articulador de los sistemas de gestión de las normas ISO 9001, ISO 14001 y OHSAS 18001, y facilita la gestión integrada en las pymes.

Para el análisis de la información recolectada en el trabajo de campo se utilizó la triangulación de diseño anidado o incrustado concurrente de modelo dominante (DIAC). El modelo predominante que guía el proyecto es el método cuantitativo y el anidado es el cualitativo; con esto se complementan los datos obtenidos y se proporciona tanto la visión de los encargados de los sistemas de gestión, como la de los auditores que participaron en el desarrollo del proyecto.

\section{RESULTADOS Y DISCUSIÓN}

En el análisis cuantitativo se encontró que la población que se encuestó considera que las pymes no realizan gestión del riesgo; en algunos casos identifican los factores internos y externos, las partes interesadas con sus 
necesidades y expectativas, el contexto donde se desarrolla la organización, los riesgos a los que se enfrenta en sus actividades y procesos, pero en general no desarrollan o contemplan la información identificada para gestionar los riesgos en las pymes. En este análisis también se determinó la confiabilidad del instrumento calculando el coeficiente alfa-Cronbach, el cual obtuvo un valor de 0,7 (aceptable).

En el análisis cualitativo de la población encuestada se evidenció que el mercado colombiano de pymes desconoce totalmente la visión de riesgo que tiene la norma ISO 31000, y el riesgo se ve y se concibe por los encargados de la gestión en la pymes únicamente a nivel de seguridad y salud en el trabajo, debido a que no tienen claridad sobre la gestión del riesgo y el concepto de riesgo establecido en la ISO 31001.

A partir de la triangulación de los hallazgos y considerando las actualizaciones de las normas mencionadas, se desarrolló un modelo de gestión que orienta a las pymes en la implementación de sistemas integrados de gestión, cuyo eje articulador será la gestión del riesgo (ver figura 1).

Figura 1. Modelo articulador de gestión del riesgo para ISO 9001, ISO 14001 y OHSAS 18001

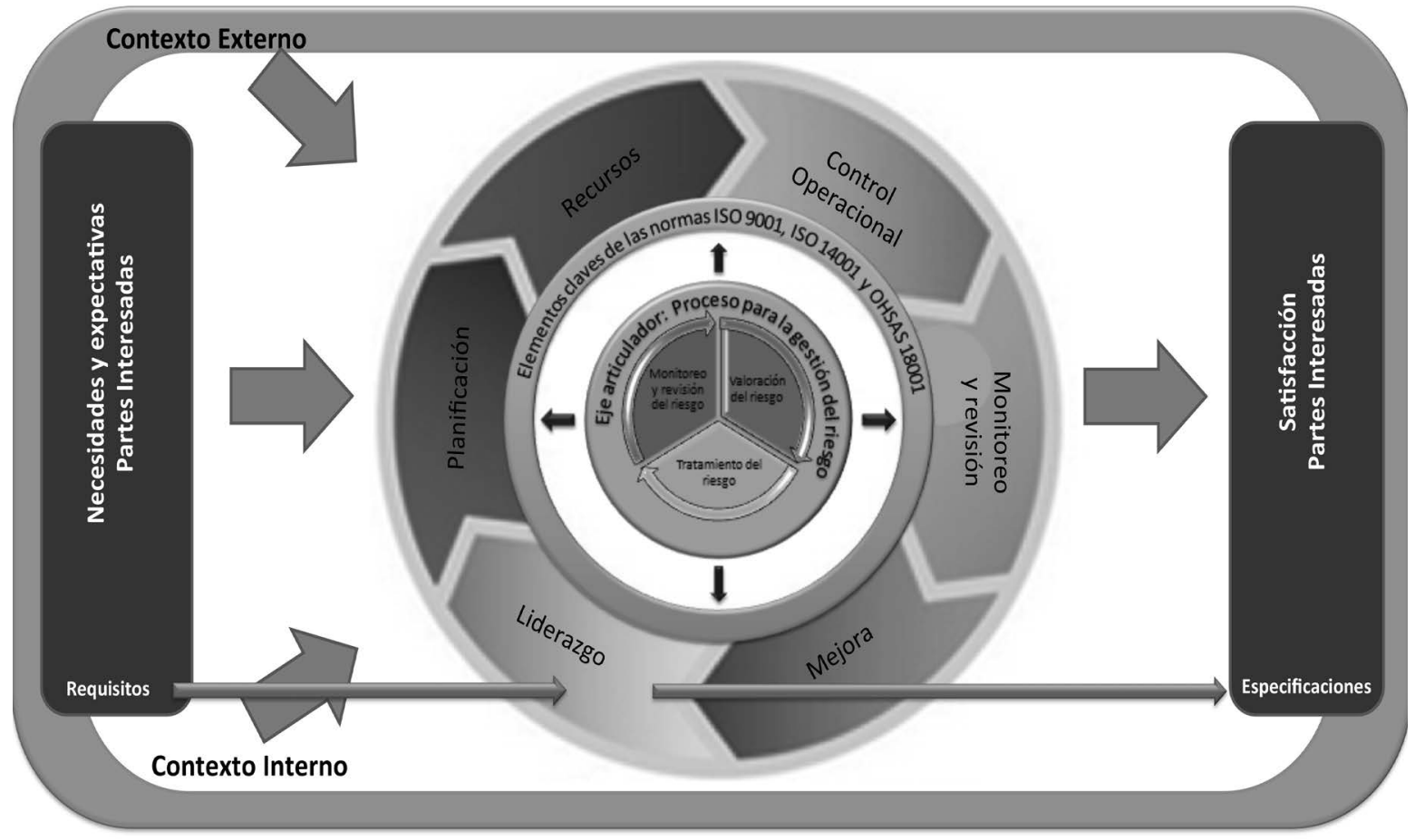

Fuente: elaboración propia. 
La clave del éxito es lograr alinear los objetivos estratégicos de la organización con los requisitos de las partes interesadas. Para facilitar la asertividad en la gestión con los grupos de interés, es clave en el proceso de identificación que las organizaciones enfoquen su análisis a las partes interesadas pertinentes, es decir, aquellas que impactan o podrían impactar los resultados esperados por la organización.

A las partes interesadas pertinentes se les identifican los requisitos específicos hacia el bien o servicio, ambiente, seguridad y salud en el trabajo, y es la organización, tomando como referencia los requisitos legales y los objetivos estratégicos, la que determina cuál de los requisitos de las partes interesadas debe cumplir. Estos requisitos son un elemento de entrada para la determinación de los riesgos.

Asimismo, es importante considerar el entorno cambiante donde se desarrollan actualmente las organizaciones, el cual tiene influencia sobre los objetivos estratégicos y políticas de la entidad. Los factores del contexto se podrían agrupar principalmente en dos categorías: interna y externa, donde las empresas tienen mayor incidencia sobre la primero y principalmente el deber de mantenerse informadas, monitorear y reaccionar ante los posibles cambios de la segundo.

Como bien se afirma, en la norma técnica ISO 9000:2005 "los líderes establecen la unidad de propósito y la orientación de la organización" (Icontec, 2005, p. ii). Los sistemas de gestión tendrán tanta utilidad y aporte a la organización como los líderes de la organización lo enfoquen. Por lo tanto, para que la gestión del riesgo sea consistente en las diferentes etapas (planear, hacer, verificar, actuar), se requiere que se encuentre determinada como una parte de las responsabilidades de la dirección (Icontec, 2011, p. 12) y que ellos asuman un rol activo, participativo, que termina demostrando la relevancia dada por la alta dirección a la gestión del riesgo en una organización. Para que a la alta dirección le sea útil la gestión, se sugiere asumir la responsabilidad frente a las actividades descritas en la Figura 2.

La alta dirección debería comprender que uno de los principios que rige la gestión del riesgo es que crea y protege el valor, y facilita la mejora continua de la organización (Icontec, 2011, p. 9); los beneficios de estos dos componentes serán retribuidos en una mejora de la productividad. Por consiguiente, los recursos que se invierten en gestión del riesgo fomentan el desarrollo organizacional. De igual manera, la alta dirección debe dar importancia a la calidad de la información, factor crítico para el éxito en la gestión del riesgo; por lo tanto, la búsqueda de la mejor información disponible debería ser promovida, apoyada y avalada por la alta dirección.

Otro elemento clave es la planificación, conocida como la gestión del riesgo que contempla la arquitectura (principios, marco de referencia y procesos) y gestionar el riesgo es la aplicación de esa arquitectura. El resultado final de una planificación es el establecimiento de objetivos y especificación de los procesos para el cumplimiento de los objetivos; esto inicia con un asertivo diseño del marco de referencia, el cual no tiene como finalidad prescribir un sistema de gestión, sino facilitar a la organización la integración de la gestión del riesgo en su sistema global (Icontec, 2011). El marco de referencia contiene diversas fuentes del riesgo, y las organizaciones, de acuerdo con sus características, podrían excluir e incluir elementos al marco de referencia.

El control operacional contempla una o más opciones para modificar los riesgos, y una vez implementados estos, el tratamiento suministra controles o los modifica. La selección de las opciones para el tratamiento del riesgo implica equilibrar los costos y los esfuerzos junto con los beneficios derivados de los requisitos legales, reglamentarios y otros. 
Figura 2. Responsabilidades de la alta dirección para la gestión del riesgo

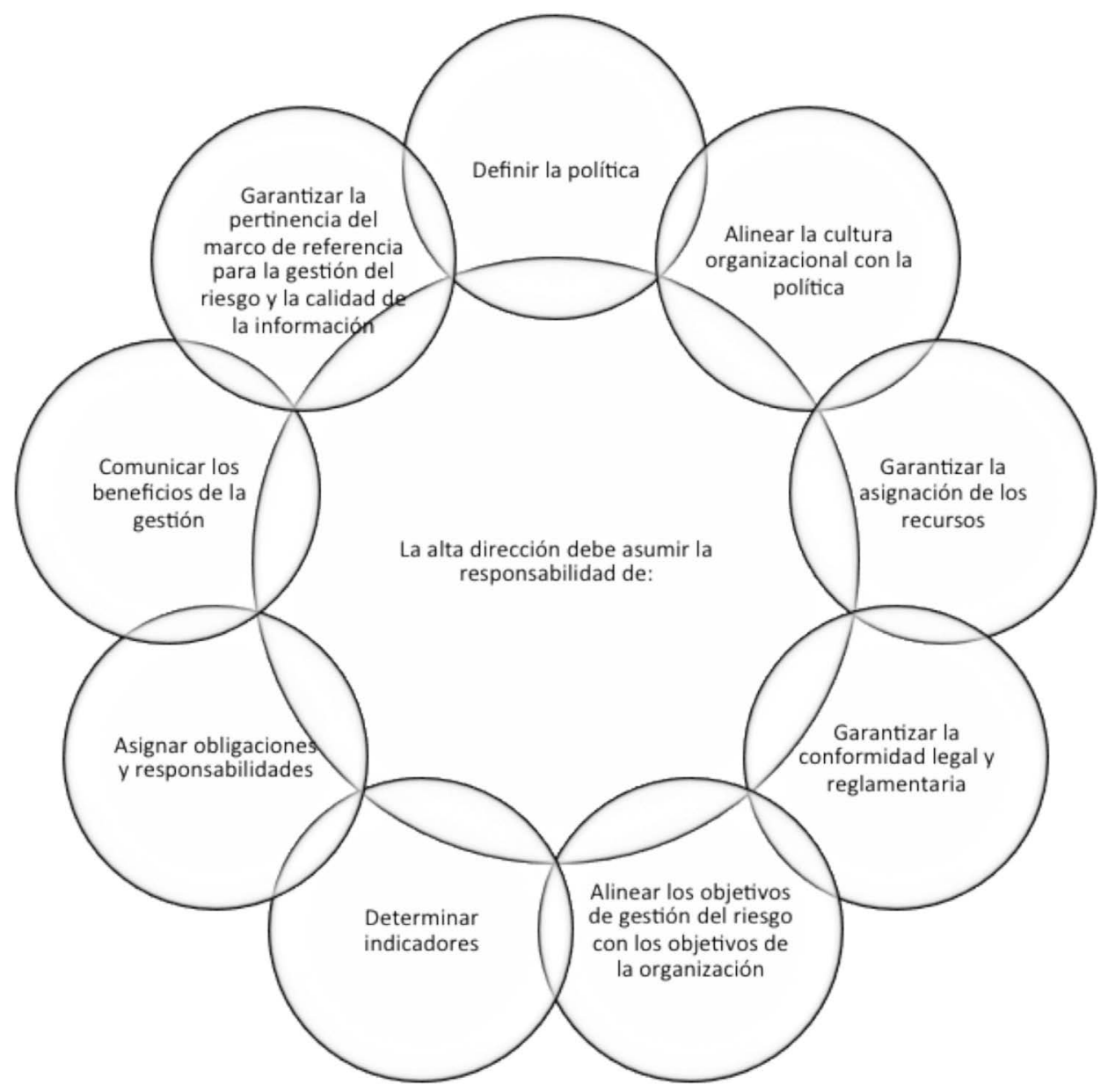

Fuente: elaboración propia a partir de Icontec (2011).

En la organización, la evaluación del riesgo debe ser un aspecto planificado. El punto de partida en la evaluación del desempeño es la definición de las responsabilidades y busca realizar seguimiento a la eficacia y eficiencia de los controles, obtener información adicional para mejorar la valoración del riesgo, lecciones aprendidas, detectar cambios en el contexto externo e interno, incluyendo cambios en los criterios del riesgo, e identificar riesgos emergentes.
La gestión del riesgo aumenta la probabilidad de alcanzar los objetivos y se reduce la probabilidad de obtener malos resultados (International Organization for Standardization [ISO], 2014); al materializarse estas dos afirmaciones, la organización manifestará de forma natural como parte de las actividades resultados de aumento de la capacidad para cumplir los requisitos. 


\section{CONCLUSIONES}

Los cambios previstos relacionados con la gestión del riesgo y la estructura de alto nivel en las versiones de las normas ISO 9001:2015, ISO 14001:2015 y Draft ISO 45001 determinan unas condiciones, que impulsan y facilitan la gestión del riesgo como eje articulador de los sistemas de gestión de calidad, seguridad, salud en el trabajo y ambiente.

En el análisis cuantitativo, el 61,5\% de los auditores 0 consultores encuestados considera que las pymes no conocen y no usan los riesgos como parte activa en la toma de decisiones; el 26,95\% afirma que sí conocen y usan los riesgos como parte activa en la toma de decisiones, pero manifiestan que este desarrollo es igual o inferior al $50 \%$. Los resultados anteriores encuentran coherencia con otros resultados: por ejemplo, en las pymes no se planifican acciones para identificar, evaluar y adoptar medidas frente a los riesgos derivados de cada proceso, $46,2 \%$ no se contempla y $53,8 \%$ se contempla, pero sin desarrollo; por consiguiente, el $76,9 \%$ afirma que las pymes no cuentan con un plan para gestionar el riesgo en todos los niveles y funciones pertinentes de la organización; el 61,5\% no cuenta con información pertinente y actualizada para identificar los riesgos, y el 34,6\% se contempla, pero sin desarrollo. Los datos presentados son consistentes con la información presentada en la descripción del problema de esta investigación, donde se afirma que las pymes enfocan sus esfuerzos en el día a día y desconocen los mercados y la competencia, entre otros.

El $100 \%$ de las personas entrevistadas de las pymes que implementan y mantienen los sistemas de gestión ISO 9001:2008, ISO 14001:2004 y OHSAS 18001:2007 manifestó no tener conocimiento de la norma ISO 31000 gestión del riesgo y asocian el término riesgo exclusivamente al campo de la seguridad y salud en el trabajo, sesgados por la norma técnica OHSAS 18001; además, realizan la gestión de los riesgos limitados al alcance establecido en dicha norma. Datos confirmados en la triangulación de la información.

La falta de claridad conceptual sobre el término riesgo en las pymes predice confusión, desconcierto y errores en el momento de implementar las versiones de las normas ISO 9001:2015, ISO 14001:2015 y Draft ISO 45001.

Con base en los resultados obtenidos para las pymes, este modelo facilita y promueve la adopción de un enfoque sistemático de la gestión del riesgo, coherente con las diferentes etapas y elementos de planificación, implementación, mantenimiento y mejora de los sistemas de gestión de calidad, seguridad, salud en el trabajo y ambiente, que lo convierte en el eje articulador y no en un componente aislado e individual, o elemento común de un sistema integrado de gestión.

El proceso y los principios de gestión del riesgo facilitan la gestión integrada en las pymes, porque la gestión del riesgo no es una actividad independiente de las labores y procesos de la organización, es parte integral de todos los procesos, de la toma de decisiones, de las responsabilidades de la dirección y la planificación estratégica. Contribuye con el logro de los objetivos y la mejora del desempeño, con un enfoque sistémico, oportuno y estructurado del sistema.

El enfoque sistemático del riesgo promueve en las organizaciones una gestión en la que prima el enfoque proactivo y preventivo sobre el reactivo y correctivo, basado en identificación, análisis, valoración, evaluación y tratamiento de los riesgos.

El establecimiento del contexto interno y externo de la organización es uno de los principales pilares para establecer un sistema de gestión integrado que sea acorde con la realidad y las necesidades de las pymes. El establecimiento de un contexto propio de la organización 
permite incluir todas las actividades y procesos, establecer objetivos, estrategias y políticas coherentes con la realidad de la organización y así facilitar la gestión integrada en las pymes. El establecimiento del contexto también incluye la identificación de las partes interesadas y de sus necesidades y expectativas, identificación que facilita la toma de decisiones, y la constitución de estrategias y objetivos que faciliten la gestión integrada.

La falta de consistencia en los resultados de la encuesta a las pymes se dio porque:

- Las empresas manifestaron sentirse prevenidas al proporcionar información para el Icontec.

- Las empresas entrevistadas manifestaron su preocupación por las actividades que no realizaban y preguntaron si esta información podía tener repercusiones sobre sus certificaciones.

- Las pymes encuestadas manifestaron que identificaban algunos factores, pero no implementaban ni profundizaban en la utilización de esta información.

- Las pymes encuestadas, en sus respuestas durante la validación de los resultados, evidenciaron un sesgo hacia el concepto de riesgo proporcionado por la OHSAS 18001 y las metodologías empleadas para manejar esta clase de riesgo.

Las pymes para implementar los elementos de gestión del riesgo, presentes en las normas ISO 9001:2015, ISO 14001:2015 y Draft ISO 45001, requieren inicialmente un cambio de mentalidad sobre riesgo del personal responsable de los sistemas de gestión y de la alta dirección en las organizaciones, susceptible de cambio en la medida que reciban formación específica en el tema de personal competente.

Es necesario ampliar el conocimiento de la gestión del riesgo en consultores, auditores, alta dirección y personal a cargo de los sistemas de gestión ISO 9001:2008, ISO 14001:2004 y OHSAS 18001:2007, con el objetivo de dejar de lado la idea de que la gestión del riesgo está únicamente ligada a la seguridad y salud en el trabajo.

De igual forma, es clave que se difunda el concepto de riesgo desarrollado en la ISO 31000 a nivel de las pymes colombianas y se dé énfasis a los beneficios que puede traer su aplicación, por su visión holística de la empresa y su aporte a la continuidad del negocio, cualquiera que sea su producto o servicio. Asimismo, es importante gestionar el riesgo en las organizaciones, en todas las actividades y procesos, para facilitar el logro de los objetivos con un enfoque preventivo, el cual permitirá una gestión integrada en la organización que proporciona una mejora en el desempeño.

Por otra parte, es necesario que los auditores sean conscientes de la importancia de la gestión del riesgo para que puedan desarrollar este tema en Colombia, como apoyo a la economía y a las pymes, en respuesta a las necesidades del país, así como a las exigencias de la economía mundial, enmarcadas en la globalización y todas las ventajas competitivas que se pueden desarrollar con una buena gestión del riesgo en el sector de las pymes.

Los elementos que conforman el modelo propuesto permiten abordar la gestión del riesgo propuesta en las normas ISO 9001:2015, ISO 14001:2015 y Draft ISO 45001; así pues, es necesario revisar cada uno de los elementos modificados y los nuevos que proponen las normas mencionadas, para garantizar el cumplimiento de la totalidad de requisitos.

El modelo propuesto en la guía pretende desplegar sistemas integrados de gestión en calidad, seguridad, salud en el trabajo y ambiente, y desarrollar así la gestión del riesgo en las etapas de planeación, implementación, verificación y mejora de los sistemas. El punto 
de partida para la organización debería ser la planeación y evitar el facilismo de desarrollarlo en una etapa de implementación sin garantizar la coherencia del modelo con el primer elemento del PHVA. El modelo es aplicable a cualquier pyme, de cualquier actividad económica, que cuente con los sistemas de gestión bajo las normas ISO 9001, ISO 14001 y OHSAS 18001, o que los desee implementar.

Para la muestra tomada en la realización de las encuestas y entrevistas, no se incluyeron variables como la competencia del personal, que permitan determinar una correlación entre el conocimiento de la gestión del riesgo y los elementos de la competencia del personal.

Los resultados de la investigación muestran un considerable porcentaje de empresas que contemplan, pero no desarrollan, elementos de la gestión del riesgo; esto se traduce en una planificación favorable, pero al mismo tiempo en una implementación deficiente, que garantice la coherencia del modelo con el actuar de la organización.

Para un desarrollo eficaz de la gestión del riesgo, la suficiencia y calidad de la información es fundamental en la construcción y actualización del modelo. Desde este modelo no se incursiona en temas relacionados con la gestión del conocimiento, aspecto fundamental para preservar el know how de los sistemas de gestión y su perdurabilidad, así como el aprendizaje organizacional.

\section{REFERENCIAS}

Álvarez, G., Roca, S., Chaux, M. y Pineda, F. (2012). Implementación del sistema de administración de riesgos (SAR) por entidades promotoras de salud del régimen contributivo en Colombia 2008-2011. Parte 1. Bogotá: Supersalud.
Atehortúa, F., Bustamante, R. y Valencia, J. (2009). Sistema de gestión integral. Una sola gestión, un solo equipo. Medellín: Universidad de Antioquia.

British Standards Institution [BSI] España (s. f.). PAS 99. Especificación de los requisitos comunes del sistema de gestión como marco para la integración. Recuperado de http://www.bsi-russia.ru/es/bsiespana/certificacion-y-auditoria/Sistemas-degestion/Biblioteca-de-BSI/Area-de-Desempeno/ LD-News-Source-/208788/

Carmona, M. (2008). La integración de sistemas de gestión normalizados sobre la base de los procesos. Estudio a partir de un panel de expertos en el ámbito del Centro Andalus para la excelencia en la gestión. Recuperado el primero de mayo de 2013, de http://excelencia. iat.es/files/2012/09/Integracion-de-SG.pdf

Carmona, M. y Rivas, M. (2010). Desarrollo de un modelo de sistema integrado de gestión mediante un enfoque basado en procesos. XIV Congreso de Ingeniería de Organización. Recuperado el primero de febrero de 2014, de http://adingor.es/congresos/ web/uploads/cio/cio2010/QUALITY_MANAGEMENT/1555-1564.pdf

Castillo, D. y Martínez, J. (2004). Enfoque para combinar e integrar la gestión de sistemas (2da. Ed.). Instituto Colombiano de Normas Técnicas y Certificación [Icontec].

Instituto Colombiano de Normas Técnicas y Certificación [Icontec]. (2005). NTC - ISO 9000 Sistema de Gestión de la Calidad. Fundamentos y vocabulario. Bogotá: autor.

Instituto Colombiano de Normas Técnicas y Certificación [Icontec]. (2011). NTC - ISO 31000 Gestión del riesgo principios y directrices. Bogotá: autor. 
Instituto Colombiano de Normas Técnicas y Certificación [Icontec]. (2012). Compendio de normas de gestión del riesgo. Bogotá: autor.

Jiménez, L. (s. f.). ¿̨Por qué necesitamos el análisis de riesgo en T.I.?. Recuperado de http://ci.ucr.ac.cr/ sites/default/files/informaciondigital/revistaci2baja.pdf

Sánchez-Toledo, A. (s. f.). Resultados esperados por una organización tras la integración de sistemas de gestión. Recuperado el 20 de abril de 2013, de http://www. mapfre.com/fundacion/html/revistas/seguridad/ n119/articulo1.html
Torres, O. y Suna, M. (2012). Del sistema integrado de gestión al sistema de gestión integral: la gestión del conocimiento como estrategia del proceso en el cuerpo de bomberos de Bogotá. Bogotá: Universidad Santo Tomas.

Velásques, M. (2012). Planificación de un sistema de gestion de la calidad como plataforma para integrar otros modelos de gestión. Bogotá: Universidad Santo Tomas.

Woods, M. (2011). Risk management in organizations. An integrated case study approach. Nueva York: Routledge. 